\title{
La experiencia de la salud y seguridad ocupacional en el Canal de Panamá
}

\author{
María Ana Molino de Antoniadis, ${ }^{1, *}$ \\ ${ }^{1}$ Ex gerente de la División de Salud Ocupacional de la Autoridad del Canal de Panamá \\ *Autor para correspondencia. Email: mantoniadis@yahoo.com
}

Recibido: 18 de marzo de 2015

Aceptado: 17 de abril de 2015

La Salud Ocupacional es un elemento importante de la Medicina Preventiva. La Organización Mundial de la Salud (OMS) la define como una actividad multidisciplinaria que promueve y protege la salud de los trabajadores y su capacidad de trabajo. Esta disciplina busca controlar los accidentes y las enfermedades mediante la reducción de las condiciones de riesgo. Es de suma importancia para los gobiernos, que deben garantizar a la población trabajadora que se cumpla con las normativas y políticas laborales y se garantice su seguridad.

El Canal de Panamá, inaugurado en 1914, juega un papel importante en el comercio mundial al unir los Océanos Atlántico y Pacífico a través de su cauce. Con el desarrollo de la globalización del comercio, el tráfico por el Canal ha aumentado significativamente en los últimos años en comparación con el que había en los años cercanos a su inauguración, cuando fue más importante el tráfico militar por las guerras mundiales que el comercial.

Desde los tiempos de la construcción del Canal, entre 1904 y 1914, la salud jugó un papel fundamental en el logro de esta portentosa obra, que ha quedado en nuestro suelo para beneficio de nuestro país y el mundo.

Gracias al saneamiento del Istmo de Panamá, con su consecuente control de las enfermedades transmisibles que, rampantes en el área acabaron con muchos nacionales y extranjeros y, en parte interfirieron con la construcción del canal por parte de los franceses, y gracias al Coronel y Dr. William C. Gorgas y sus conocimientos sobre los mosquitos como vectores de estas enfermedades, hoy el país cuenta con uno de sus más importantes activos y fuente de ingresos como es el Canal, con un control sobre enfermedades transmisibles que siguen afligiendo a nuestros vecinos, pero que aquí hemos logrado mantener bajo un mejor control.

La salud y seguridad ocupacional fueron tan importantes en esa época para proteger a los trabajadores de la construcción, como ha continuado siendo durante los 100 años de vida del Canal, hasta nuestros 
Invest. pens. crit.

Vol. 3, No. 1, enero-abril 2015.

pp. $82-86$

días. Aunque en aquellos días no había una división o unidad dedicada 100\% a estas labores y, por tanto, se dieron muchos accidentes y enfermedades, sí había actividades como el control de mosquitos, la atención médica en el hospital Ancón, más tarde llamado Gorgas, entre otras actividades.

El Dr. Gorgas fue nombrado como Jefe de Sanidad, para atender y corregir la situación sanitaria deplorable de las ciudades de Panamá y Colón, que fue considerada prioritaria antes de comenzar los trabajos. Gracias a que él había trabajado en Cuba con el Dr. Juan Carlos Finlay y la Comisión Walter Reed para aclarar el problema de la Fiebre Amarilla, una de las enfermedades que causaba estragos en Panamá al igual que la malaria, y sus relaciones con mosquitos vectores, esta hipótesis era difícil de dilucidar con los métodos de la época. A Gorgas le tomó año y medio controlar la situación en Panamá de estas dos enfermedades, además de otras también prevalentes como la tuberculosis, pulmonías e infecciones intestinales, todas enfermedades infecciosas que acabaron con miles de vidas.

Se tomaron medidas para aislar a los enfermos, fumigar, cortar los herbazales, recoger la basura y drenar las aguas estancadas que se convertían en criaderos, etc. Se construyeron acueductos y alcantarillados y se pavimentaron calles en las ciudades, inhóspitas hasta entonces, de Panamá y Colón.

La creación de la División de Salud Ocupacional en el Canal data de 1979, la cual se dedicó exclusivamente a los trabajadores de la Comisión del Canal de Panamá, como se le llamaba en ese entonces.

La ex División de Salud Ocupacional, que actualmente se llama Unidad de Salud y Bienestar Laboral, en conjunto con Seguridad e Higiene Industrial, ofrece a los trabajadores del Canal una vigilancia preventiva de su salud y seguridad en el trabajo. Estos programas son integrales y abarcan todos los aspectos necesarios para un efectivo resultado de preservar la productividad y el bienestar de sus trabajadores, lo cual obviamente redunda en beneficios para el país. También se promocionan los estilos de vida saludables y se le da seguimiento a problemas como enfermedades crónicas, abuso de alcohol y sustancias ilícitas, problemas emocionales que inciden en el trabajo, entre otros.

Las unidades de Seguridad Ocupacional e Higiene Industrial son también críticas para el logro de estos aspectos, ya que todo el trabajo se hace en equipo y colaboración. Los higienistas realizan muestreos físicos y químicos principalmente y analizan esas muestras; evalúan las hojas de seguridad de materiales, certifican trabajos en ambientes confinados o potencialmente peligrosos; evalúan la efectividad de los equipos de protección personal; educan y caminan por las áreas de trabajo. Los oficiales de seguridad también están físicamente en el campo para detectar situaciones potenciales de peligro de accidentes o incidentes; investigan accidentes, vigilan que se utilicen los equipos de protección personal, educan, corrigen derrames u otros riesgos y, en general, trabajan con los resultados del control de pérdidas. 
Invest. pens. crit.

Vol. 3, No. 1 enero-abril 2015.

pp. $82-86$

\section{El valor de tener un programa interno de salud ocupacional}

Las empresas del mundo de hoy en día saben que su capital humano es su más importante recurso. Igualmente, tener un capital humano saludable y productivo hace que las organizaciones o empresas también sean saludables y tengan éxito.

Teniendo esto presente, es fácil imaginar que lo que se invierta en mantener a los trabajadores saludables y emocionalmente satisfechos no se puede ni comparar con el costo de no hacerlo.

Cuando no se toma en cuenta el bienestar y la salud de los trabajadores, no se puede esperar que se sostenga un alto desempeño y productividad por mucho tiempo.

Por lo tanto, el tener programas internos de salud y seguridad ocupacional en las empresas garantiza la preservación de la salud de sus trabajadores y la solución de una serie de problemas que pueden afectar seriamente a la empresa, de una manera rápida, eficiente y menos costosa, en lugar de tener que diversificar externamente esa misma atención, sin controles de calidad ni el conocimiento profundo que se tiene internamente de la empresa, su cultura y sus objetivos.

En el Canal de Panamá se cuenta con programas internos de salud y seguridad ocupacional, que atienden esos fundamentales aspectos que afectan a la fuerza laboral, para lo cual, aparte de brindar un servicio de primeros auxilios en casos de enfermedades o accidentes, también se ofrece una gama de servicios como algunos de los listados a continuación:

- Una identificación y seguimiento de los riesgos a los que pudieran estar expuestos los trabajadores mediante panoramas de riesgos bien establecidos y exámenes físicos periódicos por médicos y enfermeras a las ocupaciones más arriesgadas o expuestas a peligros. Igualmente, muestreos de agentes físicos, químicos o biológicos por parte de oficiales de seguridad e higienistas industriales.

- Vigilancia y seguimiento de otras condiciones físicas, infecciosas o emocionales que pudieran interferir con el trabajo. Atención de primeros auxilios y vacunaciones en clínicas de salud ocupacional en todas las áreas operativas de trabajo, atendidas por enfermeras ocupacionales altamente capacitadas, que permiten un inmediato retorno al trabajo sin pérdida de tiempo productivo por salir del área innecesariamente.

- Educación médica preventiva y promoción de la salud por parte de los médicos, enfermeras y sicólogos, entre otros profesionales. Vigilancia epidemiológica de la entidad y de los buques en tránsito y medicina de buceo, entre otros.

- Un programa de asistencia al empleado manejado por sicólogos organizacionales, el cual brinda consejería para una gama de condiciones o situaciones que afecten el trabajo tales como las adicciones al alcohol o las drogas, el estrés laboral y personal, la violencia (intrafamiliar o en el trabajo), problemas de relaciones interpersonales, depresión, problemas económicos, estrés postraumático, mejoramiento del clima organizacional, entre otros y se ofrece "Coaching" organizacional y pruebas psicológicas para puestos sensitivos. 
Invest. pens. crit.

Vol. 3, No. 1, enero-abril 2015.

pp. $82-86$

- A los trabajadores accidentados o enfermos, se les ofrece tratamientos especiales de rehabilitación diseñados para agilizar su retorno al trabajo de manera segura y eficiente, a través de un programa de fortalecimiento para el trabajo manejado por fisioterapeutas especializados, incluyendo una reeducación para evitar recaídas. También un programa y norma de ergonomía que es muy importante tanto para posiciones de oficina como de equipo flotante.

- Asistencia de enlace con la Caja de Seguro Social y administración de un seguro privado para los trabajadores, con análisis y vigilancia de su siniestralidad, con el fin de diseñar medidas de prevención y mitigación.

- Un programa de acondicionamiento físico individual o colectivo, especialmente para las ocupaciones más exigentes físicamente, a través de gimnasios, ligas deportivas, piscinas y programas especiales. El programa de bienestar laboral ofrece actividades para mantenerse en buenas condiciones físicas, y actividades deportivas y recreativas que permiten relajar el estrés y encauzar intereses en otras cosas. También se ofrecen programas para los hijos dependientes, tanto en el verano como a través de cursos de natación y otros deportes durante todo el año. Algunas veces se motiva a que los padres participen junto con sus hijos para promover la unidad familiar. Se organizan caminatas y competencias de diversos tipos.

- No menos importante se realizan mediciones del absentismo, presentismo y otros indicadores de la gestión de salud ocupacional. Estas mediciones son muy importantes y necesarias.

- Finalmente, los trabajadores son dotados de equipos de protección personal, imprescindibles para una adecuada protección ante los diversos factores físicos, químicos o biológicos que pudieran afectar su seguridad, al igual que programas preventivos especiales para las diferentes exposiciones que pueden afectar a los trabajadores, tales como un programa de conservación auditiva, de protección respiratoria, entre otros.

Estos programas también tienen como misión, ofrecerle al trabajador las herramientas, no solamente para conservarse sano, sino también para lograr un balance adecuado de los diferentes roles de su vida. Eso es más factible hacerlo como parte de un programa interno que si se terceriza su ejecución.

\section{Evolución de la salud ocupacional}

Desde la invención de la agricultura, hasta la invención de los microprocesadores, ha aumentado mucho la capacidad de los seres humanos para producir bienes y servicios que promuevan y enriquezcan el bienestar y el disfrute de la vida.

Sin embargo, este progreso se ha acompañado de cambios en la naturaleza del trabajo que ha llevado a perder el autocontrol sobre el proceso laboral.

Como las enfermedades y accidentes relacionados con el trabajo son un producto de las condiciones bajo las cuales se trabaja, solamente al cambiar esas condiciones pueden minimizarse esas enfermedades y accidentes. 
Resulta que el trabajo es una actividad humana necesaria. La mayor parte de la gente trabaja para sobrevivir, hasta el punto de que los riesgos a la salud de sus trabajos se tornan secundarios y a menudo ignorados ante sus grandes necesidades.

Pero, la mayoría de las personas desean de sus trabajos algo más que un pago en dinero: desean recibir satisfacciones menos tangibles como la oportunidad de guiar sus propias vidas; de hacer cosas significativas; de tener interacciones sociales; el deseo de sentirse útil y deseado; y el deseo de contribuir al bien común.

Sin embargo, en otras épocas, las condiciones de trabajo dejaban mucho que desear, y fueron factores como luchas o tragedias los que abrieron paso a legislaciones y controles para mejorar esas condiciones.

Antes no se le prestaba la debida atención a los accidentes y enfermedades ocupacionales, ni a la contaminación del ambiente. Pero en 1970 salió la Ley de Salud y Seguridad Ocupacional en los Estados Unidos, la cual marcó un hito en la historia de la Salud y Seguridad Ocupacional.

La evolución que ha tenido lugar en los últimos años nos llevó desde un servicio de salud reactivo, pasando por una creciente inclinación a lo preventivo, hasta llegar a una concepción de motivación, preocupación por medición de resultados, control y mejoramiento de la calidad, resultados medibles de productividad y, en pocas palabras, un ejercicio que nos lleva a ser socios estratégicos de nuestras organizaciones.

Ahora nos interesan otras cosas: no solamente la preservación de la salud y la seguridad, sino también el bienestar físico y emocional.

En conclusión, el Canal de Panamá que se mantiene siempre a la vanguardia de muchas cosas en el país, también lo hace en este importante campo, con programas muy completos que son ejemplo para otras empresas. Antes y después de la reversión del Canal a los panameños se ha continuado dando un importante apoyo a la salud y seguridad, y se ha logrado mantener la tasa de accidentes y enfermedades a niveles inferiores que los de la industria similar. Y, a la vez, se han convertido en agentes de un menor absentismo y una mayor productividad por el bien del comercio mundial que es nuestro principal cliente $y$, por ende, por el bien del país.

\section{Referencias}

Levy, B. S., Wegman, D. H., Baron, S. L., \& Sokas, R. K. (Eds.). (2011). Occupational and environmental bealth: Recognizing and preventing disease and injury (6a. ed.). Nueva York, NY: Oxford University Press Inc.

Sánchez, G. A. (2006). El canal de Panamá y sus vidas: Vidas y muertes durante el periodo de la construcción del canal de Panamá (1904-1914) (Vol.1). Tuczon, Arizona: Hats Off Books. 